Series A

\author{
I. MATHEMATICA
}

487

\title{
KOEBE SETS FOR UNIVALENT FUNCTIONS WITH TWO PREASSIGNED VALUES
}

BY

J. KRZYŻ and E. ZLOTKIEWICZ

H E L S I K I 1971

S U O M A L A INEN TIEDEAKATEMIA

doi:10.5186/aasfm.1971.487 
Copyright (C) 1971 by

Academia Scientiarum Fennica

Communicated 9 October 1970 by Olli Lehto 


\section{INTRODUCTION}

Suppose that $M$ is a fixed, non-empty class of functions analytic and univalent in the unit disk $\Delta$. given:

In [6] the following definition of the Koebe set $\mathcal{K}(m)$ of $M$ was

$$
\mathcal{K}(m)=\bigcap_{f \in \mathcal{K}} f(\Delta)
$$

$\mathcal{K}(M)$ is not necessarily a domain in cases considered below so that the notion Koebe set rather that Koebe domain seems to be more adequate for our purposes.

If $\mathcal{S}$ denotes the class of functions $f$ univalent and normalized in the usual way: $f(0)=0, f^{\prime}(0)=1$, then obviously $\mathcal{K}(\mathcal{M})$ is Koebe's one-quarter disk.

In this paper we determine the set $\mathcal{K}(M)$ for various classes of univalent functions subject to Montel's normalization ([9], p. 66):

$$
f(0)=a, f\left(z_{0}\right)=\mathrm{b} .
$$

Some thirty years ago an analogous problem was investigated by W. W. Rogosinski [10] who gave the solution under an additional assumption of starshapedness of $f$.

In this paper we give a general and simple method of evaluating $\mathcal{K}(\mathcal{M})$ for univalent functions subject to the normalization (1.2). This enables us to reduce this problem to the following extremal problem which has an independent interest: given a point $w$ and a class $\mathscr{B}=\mathscr{S}(a, b)$ of simply connected domains $\Omega$ in the open plane $\mathscr{C}^{2}$ such that $a, b \in \Omega$, find the supremum $\mu(w,(5)$ of Green's function $g(a, b ; \Omega)$ for all $\Omega \in \mathbb{G S}$ such that $w \in \mathscr{E}^{2} \backslash \Omega$. This problem is solved here for classes of convex, starlike and close-to-convex domains (Lemmas 2-4). Also the general case of arbitrary simply connected domains is considered (Theorem 1).

As corollaries of Lemmas $1-4$ we obtain Theorems $3-5$ which yield $\mathcal{K}(M)$ for the relevant classes of functions. 


\section{BASIC LEMMAS}

We now prove the following

Lemma 1. Suppose that SS is a class of simply connected domains $\Omega$ containing two fixed, different points $a, b$ of the finite plane $\mathcal{E}^{2}$. Suppose that (S) has following properties:

(i) if for a given, finite $w$ there exists in (5) a domain omitting $w$, there exists in (S) a domain $\Omega_{w}$ with the maximal Green's function $g(a, b ; \Omega)$ among all $\Omega \in \mathbb{S}$ such that $w \in \mathscr{E} 2 \backslash \Omega$, i.e.

$$
g(a, b ; \Omega) \leq g\left(a, b ; \Omega_{w}\right)=\mu(w ; \text { (S) }
$$

for any $\Omega \in \mathbb{B S}$ such that $w \in \mathscr{E}^{2} \backslash \Omega$;

(ii) the set $\left\{z: g\left(a, z ; \Omega_{w}\right)>\delta\right\}$ belongs to (S) for all $0<\delta<g\left(a, b ; \Omega_{w}\right)$. Let $\mathbb{S S}_{\gamma}=\{\Omega \in \mathbb{B S}: g(a, b ; \Omega)=\gamma\}, \gamma>0$.

Then $\bigcap_{\Omega \in \mathfrak{B}_{\gamma}} \Omega=\{w: \mu(w, \mathbb{S})<\gamma\}$.

Proof. Suppose that $w$ does not belong to $\bigcap_{\Omega \in \mathscr{G},} \Omega$. Then there exists $\Omega_{1} \in \mathscr{F}_{\gamma}$ such that $w \in \mathscr{E}_{2} \backslash \Omega_{1}$. Now, $g\left(a, b ; \Omega_{1}\right)=\gamma \leq \mu(w)$ by $(2.1)$. Thus $\left[w \notin \bigcap_{\Omega \in \omega_{\gamma}} \Omega\right]$ implies $[\mu(w) \geq \gamma]$, or $[\mu(w)<\gamma]$ implies $\left[w \in \bigcap_{\Omega \in()_{\gamma}} \Omega\right]$.

Suppose now that $\mu(w) \geq \gamma$. From (i) it follows that $g\left(a, b ; \Omega_{w}\right)=$ $\mu(w) \geq \gamma$. Consider now $\Omega^{\delta}=\left\{z: g\left(a, z ; \Omega_{w}\right)>\delta\right\}$. Clearly $\Omega^{0}=\Omega_{w}$. Since $\gamma>0$, we have $g\left(a, b ; \Omega^{\delta}\right)=\gamma$ for suitably chosen $\delta$ with $\Omega^{\delta} \in$ BS $_{\gamma}$ by (ii). Since $\Omega^{\delta} \subset \Omega_{w}$, we have $w \in \mathscr{C}^{2} \backslash \Omega^{\delta}$ and consequently, $w \notin \bigcap_{\Omega \in \mathscr{G} \gamma} \Omega$.

This means that conversely, $\left[w \in \bigcap_{\Omega \in\left({ }_{\gamma}\right.} \Omega\right]$ implies $[\mu(w)<\gamma]$. Our lemma is proved.

We shall be now concerned with the evaluation of the function $\mu(w$, SS) for various classes of domains. We start with the class of convex domains (3) ${ }^{c}$.

Lemma 2. Let $\mathbb{S S}^{c}=\mathbb{S S}^{c}(a, b)$ be the class of all convex domains $\Omega$ containing the points $a, b$. If $w$ lies outside $[a, b], \Omega \in \mathfrak{G H}^{c}$ and $w \in \mathscr{C}_{2} \backslash \Omega$, then

$$
\begin{aligned}
\mu\left(w, \mathbb{S}^{c}\right) & =\sup _{\Omega \in \mathscr{G}^{c}} g(a, b ; \Omega)=g\left(a, b ; \Omega_{w}\right)= \\
& =\log \frac{|w-a|+|w-b|}{|a-b|}
\end{aligned}
$$

The extremal domain is a half-plane $\Omega_{w}$ whose boundary $l$ contains the point $w$ and subtends equal angles with segments $[w, a],[w, b]$.

Proof. If $\Omega$ is a convex domain containing $a, b$ and leaving $w$ 
outside, there exists a half-plane $H$ containing $\Omega$ and such that $w \in$ fr $H$. Since $g(a, b ; \Omega) \leq g(a, b ; H)$, it is sufficient to consider just the halfplanes $H$ with $w \in$ fr $H$. Suppose now that $H$ is the right half-plane, $w=0, \quad a=d e^{i \theta}, \quad b=h e^{i(\alpha+\theta)}$. Here $d, h, \alpha$ are real and fixed $(d, h>0)$, whereas $\theta$ has to be chosen so that $g\left(d e^{i \theta}, h e^{i(\alpha+\theta)} ; H\right)$ is a maximum.

We have

$$
\begin{gathered}
g\left(W, d e^{i \theta} ; H\right)=-\log |z|, \text { where } \\
z=\left(W-d e^{i \theta}\right) /\left(W+d e^{-i \theta}\right), W=h e^{i(\alpha+\theta)} .
\end{gathered}
$$

Hence $\max g\left(W, d e^{i \theta} ; H\right)$ corresponds to

$$
\begin{gathered}
\min |z|=\min _{\theta}\left|h e^{i \alpha}-d\right|\left|h e^{i(\alpha+\theta)}+d e^{-i \theta}\right|^{-1}= \\
=|a-b|(h+d)^{-\mathbf{1}}=|a-b|(|w-a|+|w-b|)^{-\mathbf{1}} .
\end{gathered}
$$

The extremal case occurs for $2 \theta=-x$, i.e. the normal of fr $H$ at $w$ bisects the angle $[a, w, b]$. Now, the equality (2.2) follows immediately from (2.3) and (2.4). A simply connected domain is called close-to-convex if it is an image domain of a disk under a close-to-convex mapping, cf. [5]. A necessary and sufficient condition for $\Omega$ to be close-to-convex is that $\mathscr{E}_{2} \backslash \Omega$ is a union of closed rays not intersecting each other [2], [8]. We say that the rays $l_{1}$ and $l_{2}$ do not intersect each other if $l_{1} \cap l_{2}$ is either empty, or it reduces to the origin of one of the rays.

We shall now evaluate the expression $\mu\left(w, \mathfrak{S}^{L}\right)$ for the class $\mathscr{S}^{L}$ of close-to-convex domains.

Lemma 3. Let $\mathfrak{S}^{L}=\mathfrak{S}^{L}(a, b)$ be the class of close-to-convex domains $\Omega$ containing the points $a, b$. If $\Omega \in \mathfrak{G}^{L}$ and $w \in \mathscr{E}_{2} \backslash \Omega$ then

$$
\begin{aligned}
& \mu\left(w, \mathfrak{S S}^{L}\right)=\sup _{\Omega \in \mathfrak{S}^{L}} g(a, b ; \Omega)=g\left(a, b ; \Omega_{w}\right)= \\
& =\frac{1}{2} \log \frac{R_{1}+R_{2}+2 \sqrt{R_{1} R_{2}}}{\left.R_{1}+R_{2}-\left[\left(R_{1}+R_{2}\right)^{2}-\mid a-b\right]^{2}\right]^{1 / 2}}
\end{aligned}
$$

where $R_{1}=|w-a|, R_{2}=|w-b|$.

The extremal domain $\Omega_{w}$ is the open plane $\mathcal{C}^{2}$ slit along a ray $l_{w}$ emanating from $w$ which subtends equal angles with segments $[w, a],[w, b]$ and does not intersect the segment $[a, b]$.

Proof. If $\Omega \in \mathbb{B S}^{L}$ and $w \in \mathscr{C}^{2} \backslash \Omega$, it follows from the geometrical definition of $\Omega$ that there exists in $\mathscr{E} 2 \backslash \Omega$ a ray $l$ containing the point $w$. On the other hand, $\Omega \subset \mathscr{E}^{2} \backslash l \in \mathscr{B S}^{L}$ and consequently, $g(a, b ; \Omega) \leq$ $g\left(a, b ; \mathscr{E}_{2} \backslash l\right)$. Hence we may restrict ourselves to the domains $\Omega=\mathscr{E}^{2} \backslash l$. By shifting $l$ along itself so that $w$ becomes its origin, 
we increase $g(a, b)$. Thus we may assume that $\Omega=\mathscr{E}_{2} \backslash l_{w}$ and $l_{w}$ is a ray emanating from $w$. We can take $l_{w}$ as the negative real axis and rotate $a, b$ round the origin, i.e. we may take $w=0, a=d e^{i \theta}$, $b=h e^{i(\alpha+\theta)}$. After a transformation $t=\sqrt{w}$ we obtain the case already considered in Lemma 2. In view of the conformal invariance of Green's function we have by $(2.2)$ :

$$
\begin{gathered}
\mu\left(w, \mathbb{S}^{L}\right)=\log \frac{\sqrt{d}+\sqrt{h}}{\left|\sqrt{d} e^{-i \alpha / 4}-\sqrt{h} e^{i \alpha / 4}\right|}= \\
=\frac{1}{2} \log \frac{d+h+2 \sqrt{h d}}{d+h-2 \sqrt{h d} \cos \alpha / 2} .
\end{gathered}
$$

In case of a maximum we may obviously assume that the ray $l_{w}$ does not intersect the segment $[a, b]$ which means that $0 \leq \alpha \leq \pi$.

Now, $\quad|a-b|^{2}=d^{2}+h^{2}-2 h d \cos \alpha=(d+h)^{2}-2 h d(1+\cos \alpha) ;$ hence $\sqrt{(d+h)^{2}-|a-b|^{2}}=2 \sqrt{h d} \cos \alpha / 2$ and finally

$$
\mu\left(w, \mathscr{B S}^{L}\right)=\frac{1}{2} \log \frac{h+d+2 \sqrt{h d}}{h+d-\sqrt{(h+d)^{2}-|a-b|^{2}}} .
$$

With $d=R_{1}, h=R_{2}$ we obtain the desired result. We can prove easily in an analogous manner

Lemma 4. Let $\mathbb{S S}^{*}=\left(\mathfrak{S}^{*}(a, b)\right.$ be the class of all domains $\Omega$ starlike with respect to $a$ and containing $b$. If $w$ lies outside $[a, b], \Omega \in \mathfrak{S}^{*}$ and $w \in \mathscr{E}_{2} \backslash \Omega$ then

$$
\begin{aligned}
& \mu\left(w, \mathscr{S S}^{*}\right)=\sup \left\{g(a, b ; \Omega): \Omega \in \mathfrak{S}^{*}, w \in^{\mathscr{C} \mathscr{E}^{2}} \backslash \Omega\right\}= \\
& =\log \left[\frac{R_{1}+R_{2}}{|a-b|}+\sqrt{\left(\frac{R_{1}+R_{2}}{|a-b|}\right)^{2}-1}\right]=\operatorname{Ar} \cosh \frac{R_{1}+R_{2}}{|a-b|} .
\end{aligned}
$$

The extremal domain $\Omega_{w}$ is the open plane $\mathscr{C g}_{2}$ slit along a ray $l_{w}$ emanating from $w$ whose prolongation contains the point $a$.

Due to symmetry of $\mu$ with respect to $R_{1}, R_{2}$ we have also

$$
\mu[w, \mathbb{S} *(a, b)]=\mu[w, \mathfrak{s} *(b, a)] .
$$

\section{AN EXTREMAL PROBLEM FOR SIMPLY CONNECTED DOMAINS}

We shall be now concerned with a counterpart of Lemmas 2-4 for general simply connected domains. We prove the following

Theorem 1. Let $\mathfrak{B S}=\mathfrak{G}(a, b)$ be the class of all simply connected domains $\Omega$ containing the points $a, b$. If $\lambda(\tau)$ is the modular function and $\tau_{0}$ is the unique solution of the equation 


$$
\lambda(\tau)=(b-a) /(w-a)
$$

contained in the fundamental domain $B$ of $\lambda(\tau)$ then the maximal value $\mu(w, \mathbb{S})$ of Green's function $g(a, b ; \Omega)$ for $\Omega \in$ (S) such that $w \in \mathscr{E}^{2} \backslash \Omega$ satisfies

$$
\mu(w, \mathfrak{S})=-\log v^{-1}\left(\frac{1}{2} \operatorname{im} \tau_{0}\right),
$$

where

$$
v(r)=\frac{1}{4} K\left(\sqrt{1-r^{2}}\right) / K(r)
$$

is the modulus of $\Delta \backslash[0, r]$. The extremal domain $\Omega_{w}$ for which the upper bound (3.2) is attained is a slit domain $\mathscr{G}_{2} \backslash C_{w}$, the slit $C_{w}$ being the image of the segment $\left[0, \frac{1}{2}\right]$ under the $\wp$-function of Weierstrass with periods $1, \tau_{0}$.

Proof. Suppose that $\Omega \in \mathbb{B}$ and $w \in \mathscr{C}_{2} \backslash \Omega$. After a suitable translation we may achieve $a+b+w=0$. Consider the family $\Gamma^{\prime}$ of all closed, rectifiable curves situated in $\Omega$ and separating $a, b$ from fr $\Omega$. It is well known that the modulus $\bmod \Gamma^{\prime}$ of the family $\Gamma^{\prime}$ satisfies

$$
\bmod \Gamma^{\prime}=v\left(e^{-g}\right)
$$

where $g=g(a, b ; \Omega)$, cf. [3].

Consider now the family $\Gamma$ of all closed, rectifiable curves separating $a, b$ from $w$ and such that the curves of both families $\Gamma^{\prime}, \Gamma$ are homotopic with respect to $\mathscr{E}_{2} \backslash\{a ; b ; w\}$. Let $\wp$ be the elliptic function of Weierstrass with periods $1, \tau(\operatorname{im} \tau>0)$ which are chosen so that $w=e_{1}=\wp\left(\frac{1}{2}\right), \quad a=e_{2}=\wp\left(\frac{1}{2} \tau\right), b=e_{3}=\wp\left(\frac{1}{2}(1+\tau)\right)$. The corresponding value $\tau$ is a solution of the equation

$$
\lambda(\tau)=\frac{e_{3}-e_{2}}{e_{1}-e_{2}}=\frac{b-a}{w-a},
$$

$\lambda$ being the elliptic modular function.

The equation (3.5) has a countable number of solutions $\tau_{k}$. There is also a countable number of homotopy classes $\Gamma_{k}$ of closed curves separating $a, b$ from $w$. If $\tau_{k}$ is a suitably chosen solution of (3.5) then

$$
\bmod \Gamma_{k}=\frac{1}{2} \operatorname{im} \tau_{k},
$$

cf. e.g. [1], p. 56.

All the solutions of (3.5) are congruent to each other with respect to the subgroup $M_{0}$ of the modular transformations $\tau^{\prime}=(a \tau+b)(c \tau+d)^{-1}$ with $a \equiv d \equiv 1(\bmod 2), c \equiv b \equiv 0(\bmod 2), a d-b c=1$.

Let $B$ be the fundamental region of $\lambda$ w.r.t. $M_{0}$, i.e. 


$$
\begin{gathered}
\operatorname{int} B=\{\tau:(\operatorname{im} \tau>0) \wedge(\mid \text { re } \tau \mid<1) \wedge \\
\left.\wedge\left(\left|\tau-\frac{1}{2}\right|>\frac{1}{2}\right) \wedge\left(\left|\tau+\frac{1}{2}\right|>\frac{1}{2}\right)\right\} .
\end{gathered}
$$

To get $B$ we add that part of fr $B$ where re $\tau \leq 0$ and im $\tau>0$. There exists a unique solution $\tau_{0}$ of (3.5) contained in $B$, cf. [4], p. 176 . The subgroup $M_{0}$ is generated by the transformations

$$
T_{0}=\tau+2, T_{1}=\tau /(1-2 \tau),
$$

cf. ibid., p. 176. The transformation $T_{0}^{k}$ (or $T_{0}^{-k}=\left(T_{0}^{-1}\right)^{k}$ ) gives for a suitably chosen integer $k$ a point $\tau$ with $\mid$ re $\tau \mid \leq 1$ and does not change $\operatorname{im} \tau$.

Hence we may consider only those $\Gamma_{k}$ which correspond to $\mid$ re $\tau_{k} \mid \leq 1$. Suppose now that $\tau$ lies in the strip $\mid$ re $\tau \mid \leq 1$ outside $B$, i.e. $\tau$ satisfies one of the inequalities $\left|\tau \mp \frac{1}{2}\right| \leq \frac{1}{2}$. Then the point $\tau^{\prime}=\tau(1 \mp 2 \tau)^{-1}$ lies in $B$, whereas im $\tau^{\prime}=|1 \mp 2 \tau|^{-2} \operatorname{im} \tau \geq \operatorname{im} \tau$. Thus among all $\tau_{k}$ which satisfy (3.5) the point $\tau_{0}$ with maximal imaginary part can be taken as the unique solution of (3.5) contained in $B$. We have $\Gamma^{\prime} \subset \Gamma=\Gamma_{k}$ for some $k$, hence by (3.4) and (3.6)

$$
v\left(e^{-g}\right)=\bmod \Gamma^{\prime} \leq \bmod \Gamma \leq \max _{k} \bmod \Gamma_{k}=\frac{1}{2} \operatorname{jm} \tau_{0}
$$

which implies $e^{-g} \geq v^{-1}\left(\frac{1}{2} \operatorname{im} \tau_{0}\right)$, or

$$
g=g(a, b ; \Omega) \leq-\log v^{-1}\left(\frac{1}{2} \operatorname{im} \tau_{0}\right),
$$

for any simply connected domain $\Omega$ with $w \in \mathscr{E}^{2} \backslash \Omega$. We now construct an extremal domain $\Omega_{w}$ for which the sign of equality in (3.8) is attained.

Given the points $a, b, w$ with $a+b+w=0$ (which may be achieved after a suitable translation), we find the solution $\tau_{0}=s_{0}+i t_{0} \in B$ of the equation (3.5).

The function

$$
u=\exp 2 \pi i \zeta=\exp 2 \pi i(\xi+i \eta)
$$

maps the parallelogram $P=\left[0,1,1+\tau_{0}, \tau_{0}\right]$ whose sides $\left[0, \tau_{0}\right]$, $\left[1,1+\tau_{0}\right]$ are identified onto the annulus $A=\left\{u: \exp \left(-2 \pi t_{0}\right)<|u|\right.$ $<1\}$. The points $\zeta=\frac{1}{2} ; \frac{1}{2} \tau_{0} ; \frac{1}{2}\left(1+\tau_{0}\right)$ correspond to $u=-1$; $\exp \left(\pi i \tau_{0}\right) ;-\exp \left(\pi i \tau_{0}\right)$, resp. We take now $r \in(0,1)$ such that

$$
v(r)=\frac{1}{2} \operatorname{im} \tau_{0}=\frac{1}{2 \pi} \log e^{\pi t_{0}}
$$

and map the ring domain $\Delta \backslash[0, r]$ conformally onto the annulus $A_{1}=$ $\left\{u: \exp \left(-\pi t_{0}\right)<|u|<1\right\} \quad$ so that $z=0, r$ correspond to $u=$ $\exp \left(\pi i \tau_{0}\right),-\exp \left(\pi i \tau_{0}\right)$, resp. The points of $A_{1}$ correspond to the lower half of $P$ in the $\zeta$-plane. If we identify in $\Delta \backslash[0, r]$ the opposite edges 
of the slit $[0, r]$ which corresponds to the identification of points on $\left[\frac{1}{2} \tau_{0}, 1+\frac{1}{2} \tau_{0}\right]$ symmetric with respect to $\frac{1}{2}\left(1+\tau_{0}\right)$ then the resulting transformation

$$
z \rightarrow \zeta \rightarrow W=\wp\left(\zeta ; 1, \tau_{0}\right)=\wp(\zeta)
$$

maps $1: 1$ conformally the unit disk $\Delta$ onto the $W$-plane slit along the arc $C_{w}$ where $C_{w}$ is the image of $[0,1]$ under $\wp(\zeta)$. Obviously the images of $\left[0, \frac{1}{2}\right],\left[\frac{1}{2}, 1\right]$ under $\wp$ are identical and equal $C_{w}$.

The fact that $\wp$ is an even elliptic function of order 2 implies the univalence of the resulting mapping, also cf. [7] p. 47. The points on $|z|=1$ corresponding to $u=1,-1$ and $\zeta=0, \frac{1}{2}$, resp. give the end-points of $C_{w}$ in the $W$-plane, i.e. the points $\wp(0)=\infty, \wp\left(\frac{1}{2}\right)=w$, whereas $z=0 \leftrightarrow u=\exp \left(\pi i \tau_{0}\right) \leftrightarrow \zeta=\frac{1}{2} \tau_{0} \leftrightarrow W=\wp\left(\frac{1}{2} \tau_{0}\right)=a$, $z=r \leftrightarrow u=-\exp \left(\pi i \tau_{0}\right) \leftrightarrow \zeta=\frac{1}{2}\left(1+\tau_{0}\right) \leftrightarrow W=\wp\left(\frac{1}{2}\left(1+\tau_{0}\right)\right)=b$.

The family of closed curves situated in $\Delta$ and separating $0, r$ from fr $\Delta$ has the modulus $v(r)=\frac{1}{2}$ im $\tau_{0}$ according to (3.9). On the other hand, by the conformal invariance of Green's function

$$
g\left(a, b ; \mathscr{G}_{2} \backslash C_{w}\right)=g(0, r ; \Delta)=-\log r .
$$

From (3.9) and (3.11) the equality

$$
g\left(a, b ; \mathscr{C}_{2} \backslash C_{w}\right)=-\log v^{-1}\left(\frac{1}{2} \operatorname{im} \tau_{0}\right)
$$

follows by eliminating $r$.

Theorem 1 is proved.

A related extremal problem was investigated by Schiffer [11] who solved it in a different way by variational methods; also cf. [12].

\section{THE DETERMINATION OF KOEBE SETS}

Given $z_{0} \in \Delta$ consider the class $9 n=9 n\left(z_{0}\right)$ of functions analytic and univalent in the unit disk $\Delta$ which satisfy the conditions

$$
f(0)=0, \quad f\left(z_{0}\right)=1 .
$$

If $f \in M$ then $\Omega=f(\Delta) \in \mathscr{B}(0,1)$ where $\mathscr{G}(0,1)$ is the class of all simply connected domains containing 0,1 . With each $f \in M$ we can associate a domain $\Omega=f(\Delta) \in \mathscr{B S}_{\gamma}$, where $\gamma=-\log \left|z_{0}\right|$ which is an obvious consequence of the conformal invariance of Green's function.

Conversely, if $\Omega \in \mathbb{S}_{\gamma}$ then $\Omega$ can be mapped on $\Delta$ conformally so that $\varphi(0)=0, \varphi(1)=\zeta_{0}$, where $-\log \left|\zeta_{0}\right|=\gamma$. Hence $z_{0}=e^{i \beta} \zeta_{0}$ for suitably chosen $\beta$ and $\varphi^{-1}\left(e^{-i \beta} z\right) \in M$.

Thus 
where $\gamma=-\log \left|z_{0}\right|$.

$$
\bigcap_{f \in \mathcal{M}} f(\Delta)=\bigcap_{\left.\Omega \in \xi_{\gamma}\right)} \Omega
$$

The above considerations as well as Lemma 1 yield

Theorem 2. The Koebe set $\mathcal{K}(\mathcal{M})=\bigcap f(\Delta)$ for the class $9 m=9 m\left(z_{0}\right)$ $f \in \mathcal{C}$

of functions analytic and univalent in the unit disk $\triangle$ subject to the normalization (4.1) is the image set of $B \cap H(r)$ under the mapping $w=1 / \lambda(\tau)$, where $r=\left|z_{0}\right|, H(r)=\{\tau: \operatorname{im} \tau<2 v(r)\}, \quad v$ being defined by (3.3); $\lambda$ is the elliptic modular function and $B$ is its fundamental region.

The set $\mathcal{K}(M)$ is symmetric with respect to the point $w=\frac{1}{2}$ and is a simply connected Jordan domain for $0<r<2^{-1 / 2}$. For $2^{-1 / 2} \leq r<1$ the set $\mathcal{K}(M)$ is a union of two congruent, disjoint, simply connected Jordan domains.

Proof. According to our previous remarks $\mathcal{K}(\mathcal{M})=\bigcap_{\Omega \in \Theta_{\gamma}} \Omega$ with $\gamma=-\log r$. In view of Lemma 1 and Theorem $1 \bigcap_{\Omega \in \mathscr{S}_{\gamma}} \Omega=\{w: \mu(w, \mathbb{S})$ $\langle\gamma\}=\left\{w: \operatorname{im} \tau_{0}(w)<2 v(r)\right\}$. Thus $\tau_{0}(w) \in B \cap H(r)$. Moreover, $\tau_{0}(w)$ satisfies (3.5) with $a=0, b=1$, i.e. $\lambda\left(\tau_{0}\right)=1 / w$, or $w=1 / \lambda\left(\tau_{0}\right)$. This shows that $w \in \mathcal{K}(\mathcal{M})$, iff $w=1 / \lambda\left(\tau_{0}\right)$ with $\tau_{0} \in B \cap H(r)$.

We now prove the symmetry property.

To this end it is sufficient to show that $1-w_{0} \in \mathcal{K}(9)$ as soon as $w_{0} \in \mathcal{K}\left(\mathcal{M}_{)}\right)$.

Suppose that $\tau_{0} \in B \cap H(r)$ satisfies $w_{0}=\left[\lambda\left(\tau_{0}\right)\right]^{-1}$. Obviously one of the points $\tau_{0} \mp 1$, say $\tau_{1}$, also belongs to $B \cap H(r)$. Since $\lambda\left(\tau_{0} \mp 1\right)$ $=\lambda\left(\tau_{0}\right) /\left[\lambda\left(\tau_{0}\right)-1\right]=\lambda\left(\tau_{1}\right)$, we have $w_{1}=\left[\lambda\left(\tau_{1}\right)\right]^{-1}=\left[\lambda\left(\tau_{0}\right)\right]^{-1}\left[\lambda\left(\tau_{0}\right)-1\right]$ $=1-w_{0} \in \mathcal{K}(\mathcal{M})$.

Suppose now that $v(r)>\frac{1}{4}$, or $r<2^{-1 / 2}$. Then the image of $B \cap M(r)$ is a Jordan domain whose boundary has the parametric representation

$$
w=[\lambda(t+2 i v(r))]^{-1},-1 \leq t \leq 1 .
$$

If $v(r) \leq \frac{1}{4}$, or $r \geq 2^{-1 / 2}$, then the set $B \cap[-1+2 i v(r), 1+2 i v(r)]$ is a union of three segments. If $\tau, \tau_{1}$ are the end-points of the intermediate segment then $\left[\tau_{1}=\tau /(1-2 \tau)\right] \in M_{0}$ and hence $\lambda\left(\tau_{1}\right)=\lambda(\tau)$ which means that the image under $1 / \lambda$ of $\left[\tau, \tau_{1}\right]$ is a closed Jordan curve. Similarly the images of the remaining two segments set up a congruent Jordan curve. Theorem 2 is proved.

A slightly more general case of functions with normalization (1.2) reduces to the case just considered by the transformation $W=(w-a) /$ $(b-a)$.

We now apply Lemma 1 in a similar way as before with $\mathscr{B S}=\mathscr{H}^{c}$, $\mathscr{B S}^{\prime}, \mathfrak{B S}^{L}$ resp. and obtain in view of Lemma $2-4$ the following theorems. 
Theorem 3. Let $m^{c}\left(z_{0}, a, b\right)$ be the class of all convex mappings $f$ of the unit disk $\Delta$ subject to the normalization (1.2). The Koebe set $\mathcal{K}\left[M^{c}\left(z_{0}, a, b\right)\right]$ is the ellipse

$$
\left\{w:|w-a|+|w-b|<|a-b| /\left|z_{0}\right|\right\} .
$$

Theorem 4 [10]. Let $9 *\left(z_{0}, a, b\right)$ be the class of all mappings $f$ of the unit disk $\Delta$ subject to the normalization (1.2) and starlike with respect to $a$. The Koebe set $\mathcal{K}\left[\mathcal{G} \cap *\left(z_{0}, a, b\right)\right]$ is the ellipse

$$
\left\{w:|w-a|+|w-b|<\frac{1}{2}|a-b|\left(\left|z_{0}\right|+\left|z_{0}\right|^{-1}\right)\right\} .
$$

Theorem 5. Let $M^{L}\left(z_{0}, a, b\right)$ be the class of all close-to-convex mappings of the unit disk $\triangle$ subject to the normalization (1.2). The Koebe set $\mathcal{K}\left[\mathcal{M}^{\mathrm{L}}\left(z_{0}, a, b\right)\right]$ has the form

$$
\left\{w: \frac{R_{1}+R_{2}+2 \sqrt{R_{1} R_{2}}}{R_{1}+R_{2}-\left[\left(R_{1}+R_{2}\right)^{2}-|a-b|^{2}\right]^{1 / 2}}<\left|z_{0}\right|^{-2}\right\}
$$

where $R_{1}=|w-a|, R_{2}=|w-b|$.

Obviously $\mathcal{K}\left(M^{\mathrm{L}}\right)$ has $w_{0}=\frac{1}{2}(a+b)$ as a centre of symmetry. Moreover, $w_{0} \in \mathcal{K}\left(M^{L}\right)$ iff $\left|z_{0}\right|<2^{-1 / 2}$. It is easily verified that for $\left|z_{0}\right|<2^{-1 / 2}$ the set $\mathcal{K}\left(9 M^{L}\right)$ is a Jordan domain and for $\left|z_{0}\right| \geq 2^{-1 / 2}$ it is a union of two disjoint Jordan domains containing $a$ and $b$, resp.

We conclude with an interesting consequence of Theorem 2 . Since the reflections with respect to the real axis and the straight line through $0, z_{0}$ yield again a mapping of the class $9 n$, we see that the real axis, as well as the line re $w=1 / 2$ are lines of symmetry of $\mathcal{K}(7)$. This implies that $[0,1] \subset \mathcal{K}(m)$, if $0<z_{0}<2^{-1 / 2}$. Hence we deduce the following Conollary. If $f$ is regular and univalent in the unit disk $\triangle$ and $\mathrm{z}_{1}, \mathrm{z}_{2} \in \triangle$ are such that the hyperbolic distance $\mathrm{h}\left(\mathrm{z}_{1}, \mathrm{z}_{2}\right)<$ ar tanh $\left(2^{-1 / 2}\right)$ then the image domain $f(\triangle)$ contains the straight line segment with end points $\mathrm{f}\left(\mathrm{z}_{1}\right), \mathrm{f}\left(\mathrm{z}_{2}\right)$. The constant ar tanh $\left(2^{-1 / 2}\right)$ is best possible.

M. Curie - Skłodowska University

Lublin, Poland 


\section{References}

[1] Ahlfors, L. V.: Lectures on quasiconformal mappings - Princeton 1966.

[2] Biernacki, M.: Sur la représentation conforme des domaines linéairement accessibles - Prace Mat. Fiz. 44 (1936), $293-314$.

[3] Hersch, J.: Longeurs extremales et théorie des fonctions - Comment. Math. Helv. 29 (1955), $301-337$.

[4] Hille, E.: Analytic Function Theory, vol. II - Boston 1962.

[5] Kaplan, W.: Close-to-convex schlicht functions - Michigan Math. J. 1 (1952), $169-185$.

[6] Krzyż, J. and Reade, M. O.: Koebe domains for certain classes of analytic functions - J. Analyse Math. 28 (1967), 185-195.

[7] Lehto, O. und Virtanen, K. I.: Quasikonforme Abbildungen - Berlin-Heidelberg-New York 1965.

[8] Lewandowski, Z.: Sur l'identité de certaines classes de fonctions univalentes I Ann. Univ. Mariae Curie-Skłodowska Sect. A 12 (1958), 131-146.

[9] Montel, P.: Leçons sur les fonctions univalentes on multivalentes - Paris 1933.

[10] Rogosinski, W. W.: Über den Wertevorrat einer analytischen Funktion, von der zwei Werte vorgegeben sind - Compositio Math. 3 (1936), 199-226.

[11] Schiffer, M. M.: On the modulus of doubly-connected domains - Quart. J. Math., Oxford Ser. 17 (1946), $197-213$.

[12] W Іттісн, H.: Über eine Extremalaufgabe der konformen Abbildung - Arch. d. Math., $2(1949 / 50), 325-333$. 